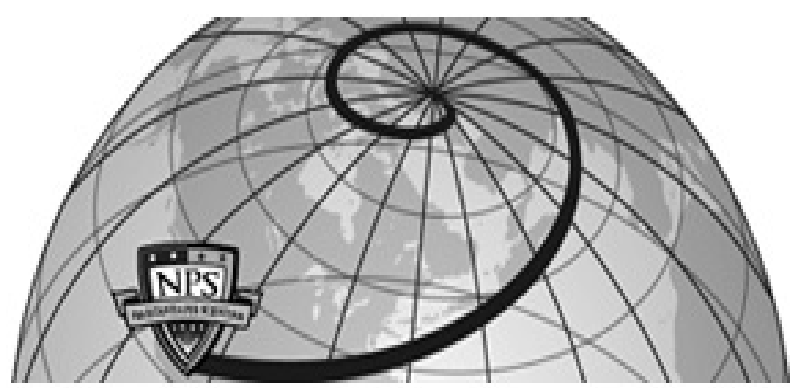

Calhoun: The NPS Institutional Archive DSpace Repository

Parametric Models for Aircraft Engine Removals Resulting from Foreign Object Damage

Millar, Richard C.; Olwell, David H.

Naval Engineers Journal

https://hdl.handle.net/10945/43488

This publication is a work of the U.S. Government as defined in Title 17, United States Code, Section 101. Copyright protection is not available for this work in the United States.

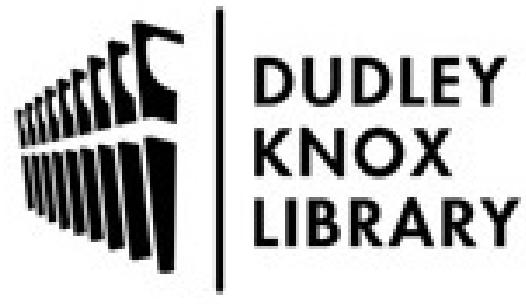

http://www.nps.edu/library
Calhoun is the Naval Postgraduate School's public access digital repository for research materials and institutional publications created by the NPS community. Calhoun is named for Professor of Mathematics Guy K. Calhoun, NPS's first appointed -- and published -- scholarly author.

Dudley Knox Library / Naval Postgraduate School 411 Dyer Road / 1 University Circle Monterey, California USA 93943 


\title{
Parametric Models for Aircraft Engine Removals Resulting from Foreign Object Damage
}

\author{
Richard C. Millar and David H. Olwell
}

\begin{abstract}
An earlier nonparametric statistical study of GE F414 engine removals from operational F/A-18 aircraft in US Navy service provided insights into the lifetime patterns of engine removals for various causes. Inspection of the estimated hazard function for engine removals for foreign object damage (FOD) suggested that a parametric analysis using Erlang distributions might be fruitful, bolstered by a hypothesized relevance to the maintenance procedures governing engine removals for this cause, and their outcomes. The objective was both a better model to forecast engine removals and to provide insight into the number of FOD incidents it took to drive an engine removal. Gamma and Erlang distributions did better fit the removals data and provide a tool for predicting engine removals, aircraft availability impact, and the resultant maintenance workload. A parametric model using a cascade of Erlang functions was developed to simulate the combined FOD/line maintenance process, which provides insight into the outcomes expected under reasonable simplifying assumptions. This model predicts that the key research issue, the probability that a typical FOD event prompts a removal, cannot be estimated from engine removals data alone. Field data must be collected to gain understanding of the underlying frequency of FOD and the utility of the present inspection criteria.
\end{abstract}

\section{Introduction}

In earlier papers Millar et al. (2009) and Millar et al. (2007) report the results of a nonparametric statistical study of unscheduled engine removals data from records of operational service of the F/A-18 E/F Super Hornet, powered by a pair of General Electric F414 low bypass gas turbine engines. Engine removals are likely the most disruptive and costly maintenance action affecting naval aircraft, particularly if unscheduled on board an aircraft carrier in action at sea. The aircraft must be removed from flight operations to remove and replace the affected engine, the largest piece of equipment that can be swapped out this way. The affected engine is usually shipped to an intermediate maintenance base and a replacement spare engine is usually delivered to the ship. The engine is inspected and possibly tested at the intermediate maintenance base, and any modules requiring teardown for detail inspection and repair are replaced with spare modules. The modules to be serviced are forwarded to a maintenance depot to be rebuilt.

Good understanding of the likelihood of unscheduled engine removals as a result of periodic inspection or damage detected in service is needed to optimize maintenance planning, facilities and manning, logistics, and spares and parts provisioning. The purpose of this and the earlier studies was to characterize the pattern of engine removals for various causes as a function of accumulated engine operating hours. This information enables maintenance and logistics 
planning for unscheduled engine removals based on the history of the specific engines in service at a given location. For example, the probability of having to remove an engine from a specific aircraft due for inspection, for a specific reason for removal, can be better forecast with the methods developed during this project, based on the accumulated operating hours of the specific engines involved. Planning preventive and corrective maintenance over a longer horizon can be based on a firmer forecast of removals and the likely causes. Personnel, tools and equipment, logistics, and spares can be provisioned in advance to speed turnaround and minimize costly spares stocks.

Furthermore, improved characterization of the likelihood of engine removals is central to the deployment of condition-based maintenance (CBM) informed by reliability centered maintenance (RCM), known as $\mathrm{CBM}+$ in the Department of Defense (US Department of Defense 2007) and a key tenet of naval aviation maintenance (NAVAIR 00-25-403 2005).

The earlier study yielded estimates for the hazard rate and survivor function through to overhaul for three classes of engine reasons for removal. The data used aggregated records from all F414 engines installed in F/A-18 aircraft over the first 8 years of US Navy operational service. More detail on this study can be found in Millar (2007).

One of the leading classifications of reasons for removal was confirmed to be foreign object damage (FOD) to the engine fan or compressor, as detected through engine failure, pilot "squawk," or, most commonly, during periodic inspection at a more or less fixed interval. FOD alone caused about $20 \%$ of all unscheduled engine removals. The other two classes of reasons for removal combined multiple reasons for removal, had different statistical characteristics, and were considered to be qualitatively different in being more dependent on inherent engine component reliability and maintenance processes rather than exogenous influences.
FOD may result from bird ingestion in flight or, more commonly, it is due to debris sucked into the engines during operation on the ground (including shipboard) or during takeoff and landing. The exposure of individual aircraft to these flight conditions and thus FOD hazard is variable depending on operational location and mission. Over a large population of aircraft performing similar missions in a variety of environments, given the lack of detailed information on the exposure and usage on individual aircraft, our basic expectation was that aggregate FOD hazard levels should not vary greatly over the engine lifetime, i.e., FOD incidents and resultant removals might be expected to follow an exponential distribution.

The second factor that affects the incidence of engine removals for FOD is the severity of FOD damage in terms of continued safe and reliable engine operation. Each FOD incident (ingestion of a foreign object capable of causing damage) may result in many instances of FOD to hardware throughout the fan and compressor modules. The severity of FOD varies widely as a result of the diversity of objects ingested, and the need for engine removal is also affected by the location of the FOD. Apparently minor damage that might elsewhere be ignored or dressed out may be a cause for removal if it affects a highly stressed location on a fan or compressor blade or vane.

The F414-GE-400 Intermediate Maintenance Manual (Anon 2009) is typical in its treatment of FOD, categorizing specific instances of FOD (nicks, dents, scratches, cracks, tears, curling, burrs, etc.) according to location (platform, shroud, blade surfaces, blade size, leading or trailing edge, blade tips, etc.). FOD size and number limits are then specified and used to determine allowable corrective action and repairable limits. The line maintenance (O-level) fan and compressor inspection and repair instructions are reported to be similar in format and usage (a copy of the O-level instructions was not available for study). These instructions provide criteria for each category of damage, criteria that determine if the damage is 

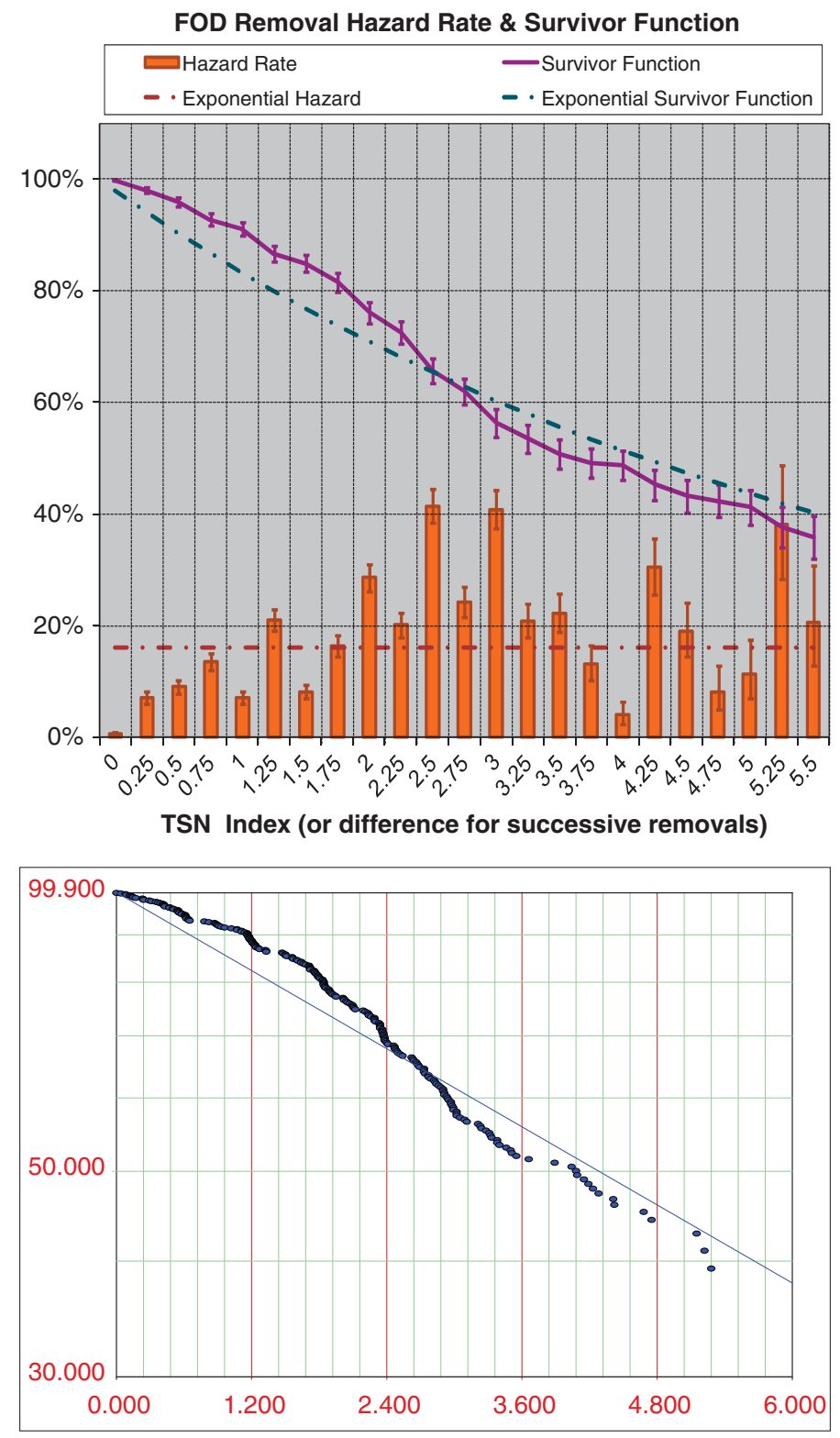

Figure 1: Estimated Hazard Rate and Survivor Function: F414 FOD Removals and Exponential Fit. Taken from Millar et al. (2009). TSN, Time Since New in Engine Flight Hours (EFH). The presentation on the top "restarts the clock" after each FOD removal; it represents the number of removals in proportion to the average population scaled to a rate per unit time. Note the $90 \%$ confidence intervals on the proportions and the cumulative data points. The results of an exponential fit (dashed lines, $\lambda=0.1618$ ) to the FOD removals data is added for comparison to the life table estimates. The correlation coefficient is 0.8475 . A "probability plot" of this fit is shown below. acceptable as is, can be mitigated by in situ repair, or requires removal of the engine for return to a maintenance facility for removal and replacement of the affected module.

The intent of these maintenance procedures is to allow field repair of nonlife-limiting damage-at O-level while installed in the aircraft to allow the aircraft to return to service without engine removal and replacement. Unfortunately the available maintenance data does not record if and when an engine was successfully repaired while installed in the aircraft, and thus how many times this occurred before an actual FOD removal, so the data analyzed recorded only removals due to FOD from one or more FOD incidents before scheduled or unscheduled inspection.

Figure $\mathbf{1}^{1}$ illustrates the original life table-based estimates of hazard rate and survivor function

\footnotetext{
${ }^{1}$ The TSN index is normalized for public release using an arbitrary EFH interval.
} 
Figure 2: Erlang Distribution $(\kappa=2, \lambda=0.43$ ) Compared with Life time Estimates of F414 FOD Removals. In this comparison an integral shape factor $\kappa=2$ appeared to be more suitable than $\kappa=1$ (an exponential distribution) or $\kappa=3$.

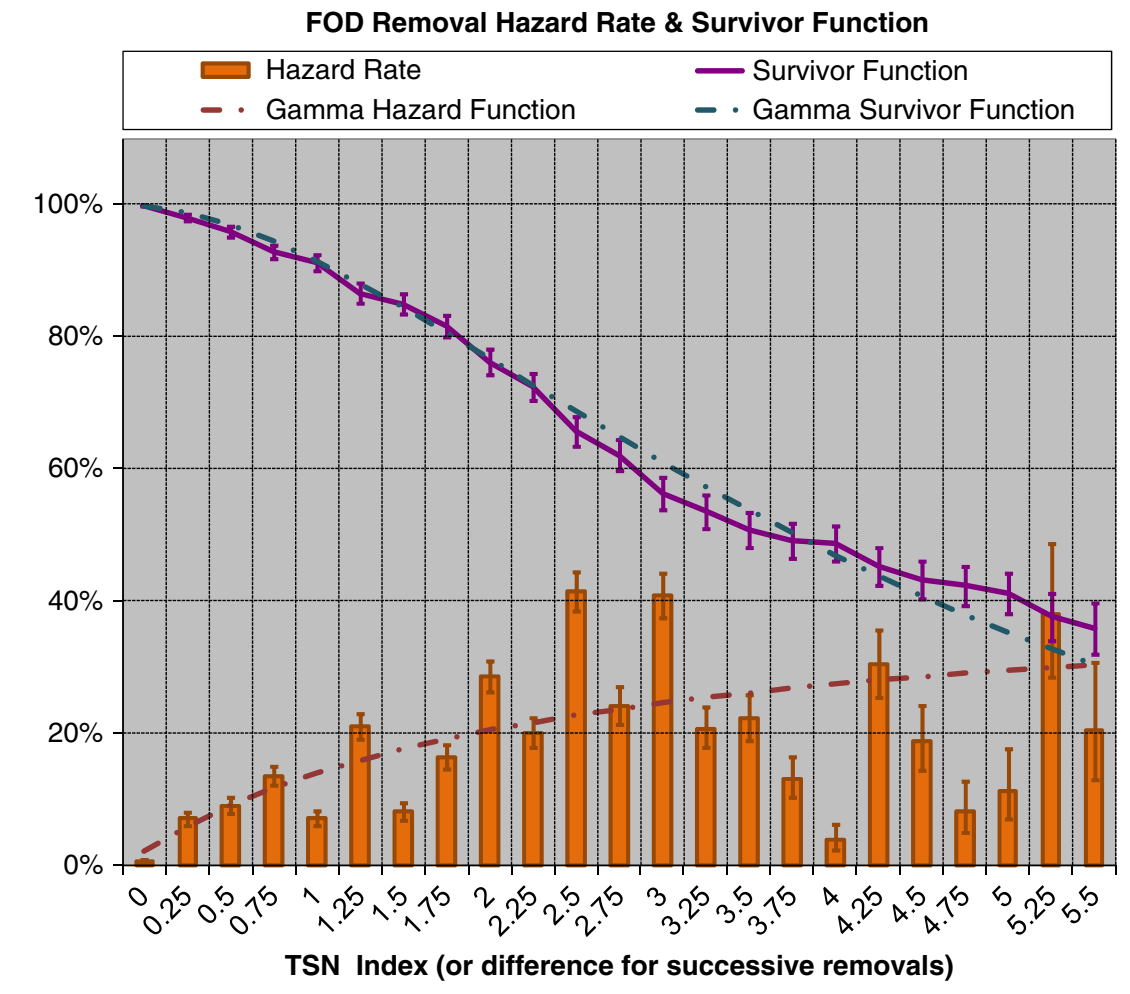

FOD Removal Hazard Rate \& Survivor Function

for FOD removals, and compares them to those of an exponential distribution fit to the FOD removals data. ${ }^{2}$ Given the complexity of the total process leading to FOD removals, the match to an exponential distribution was considered reasonable, and at least provides a basis for planning FOD-related engine removals. There was a roughly constant $9 \%$ probability of an engine removal for FOD during a scheduled inspection interval, independent of accumulated engine flight hours, and most engines were pulled for FOD at least once before overhaul.

Other notable features include no evidence of periodicity, although most FOD removals occur as a consequence of periodic inspections, and the gradual initial build up of the hazard function. It is hypothesized that discretion in the timing of inspections to accommodate operational exigencies may wash out the impact of the former, and that the latter may be an artifact of the initial hiatus

${ }^{2}$ Parametric fits to the data presented here were performed using Reliasoft Weibull++. There were 238 failure and 426 suspense data points. until the first inspection plus the possibility of in situ FOD rework, discussed above, allowing the avoidance of many early removals.

However, the question arises: what might be a better distribution to fit to this data and what implications does this have for our understanding and management of line maintenance for this engine failure mode? This implies application of statistical tools to model the combination of a stochastic damage process and a defined maintenance procedure.

\section{Inspiration and Exploration GAMMA DISTRIBUTION PROPOSAL}

On reviewing the life table hazard estimates distribution, Dr. Olwell noted that the shape of the estimated hazard distribution might be well represented by a Gamma function. This was an intriguing suggestion in this specific context due to the well-known result (e.g., see Lawless 2003, section 1.3.5) that Gamma distributions with integral shape parameter (i.e., $\kappa=1,2, \ldots n$ )otherwise, and below, referred to as Erlang distributions-are equivalent to the cumulative 
time of $n$ exponential random variables with the same scale parameter, i.e., " $T 1+T 2+\ldots+T n$ has a gamma [Erlang] distribution” (Lawless 2003).

The crucial insight was that FOD might be a good analog to this scenario, because of the expectation that it might take more than one FOD incident to result in an engine removal. Our hypothesis was that it might be possible to estimate the average number of FOD incidents needed to "drive an engine off-wing" by fitting an Erlang distribution to the F414 FOD removal data and thus develop a parametric estimate of the total FOD incidence. Figure 2 illustrates this approach, where a shape factor of 2 could be interpreted as an indication that exactly two FOD incidents resulted in an engine removal. However, unless we postulate a consistent practice of doing this, which contradicts all other information available, this seems unlikely.

The obvious next step was to fit a Gamma distribution to the empirical data, as illustrated in Figure 3. Qualitatively this result may indicate that the average number of FOD incidents before an engine needs to be removed might be somewhat less than two, however, a Gamma distribution with a given $\kappa$ and $\lambda$ cannot be readily decomposed analytically into a mix of Erlang $(\kappa=1,2,3, \ldots)$ distributions that might suggest a distribution for the number of FOD incidents before engine removal.

Pragmatically, either the Erlang or Gamma distribution provides a better tool than the exponential for data driven models to predict the impact of "time on wing" on aircraft availability and line maintenance planning, i.e., removals for FOD are significantly less likely during early scheduled inspections but may be more than twice as high later.

However, due to the variability in characteristics of FOD, it seems likely that the FOD removals data is indeed a mix of removals after one, two, three, or more FOD hits on individual engines.
Thus, it seems a simple Erlang/Gamma parametric fit to the data may be a useful distribution for modeling the end result, the rate of removals as a function of engine operating hours, but is not a very informative model to help us understand the underlying FOD/inspection/removal process.

\section{MODELING FOD AND THE MAINTENANCE PROCESS}

A model is needed to help understand the total process driving engine removals for FOD, i.e., a simulation of the accumulation of FOD, inspection/detection, and removal for excessive damage. A Gamma distribution gives an excellent fit to the removals data, but does not simulate the maintenance process. The above results could not disprove the presumed exponential FOD incident hazard rate for the underlying FOD incidence, so our simulation model started with that basic assumption, making the further simplifying assumption that the scale factor $(\lambda)$ of the FOD distribution will not change for successive hits, as there was no obvious and plausible rationale for such a dependence.

To model the process of FOD maintenance, it seemed reasonable to assume that the probability of removal after a FOD incident $\left(P_{\mathrm{R}}\right)$ is also a constant, on average, independent of whether it is the first, second, or later incident. This implies that subsequent FOD severity is unaffected by prior damage, e.g., a small probability that overlapping damage fields aggravate damage severity.

These assumptions led to a process model consisting of a cascade of Erlang distributions, with $\kappa=1,2,3$, and on, representing removals following one, two, three, or more FOD incidents, respectively. If the probability of removal after each FOD incident is $P_{\mathrm{R}}$, the contribution of $\kappa=1$ to removals would be $P_{\mathrm{R}}$ times the Erlang distribution with $\kappa=1$ (an exponential), that of $\kappa=2$ would be $P_{\mathrm{R}}\left(1-P_{\mathrm{R}}\right)$ times the Erlang of $\kappa=2$, that of $\kappa=3$ would be $P_{\mathrm{R}}\left(1-P_{\mathrm{R}}\right)^{2}$ times the Erlang of $\kappa=3$, etc. 
Figure 3: Gamma Distribution $(\kappa=1.7133, \lambda=0.3619)$ Fit Compared with Life time Estimates of F 414 FOD Removals. The probability plot at the bottom of the fit to the empirical removals was produced with Weibull++. The correlation coefficient is 0.9965 . This is clearly preferable to the exponential fit in Figure 1.
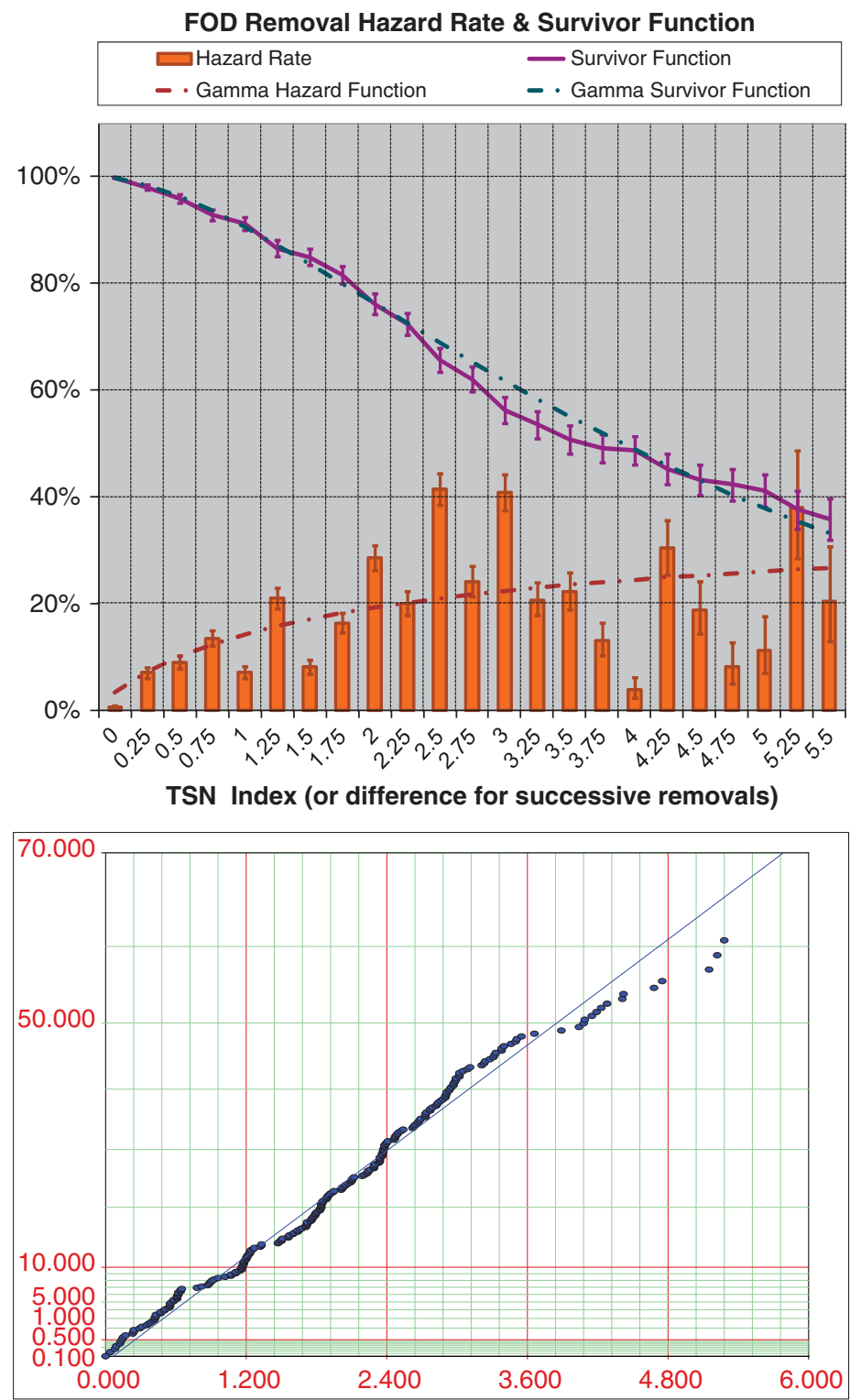

This series rapidly converges with increasing $\kappa$, as shown in Table 1, to a sum that numerically equals an exponential distribution for FOD removals with a failure rate $=\left(\lambda \times P_{\mathrm{R}}\right)$. In retrospect, this result is obvious since each FOD event has a probability of $P_{\mathrm{R}}$ of causing a removal, resulting in a hazard rate for FOD removals equal to $P_{\mathrm{R}}$ times the underlying FOD hazard rate. Figure 4 illustrates this convergence, based on a $50 \%$ removal probability after a FOD incident and the 0.1618 FOD removal hazard rate corresponding to the exponential fit in Figure 1.
The unexpected implication from this model, given the simplifying assumptions, is that the sum of this attenuated series of Erlang distributions is an exponential distribution, as evidenced in the last two rows of Table 1, thus predicting that engine removals should also be exponential. Furthermore, we can estimate neither $P_{\mathrm{R}}$ nor the underlying FOD rate $\left(\lambda_{\mathrm{FOD}}\right)$ from observing their product, $\lambda_{\mathrm{FOD}}$ Removal. Thus our original objective, to estimate $P_{\mathrm{R}}$ from the observed FOD removals, is impossible if this model is representative. Thus we have a process model simulating FOD plus the associated maintenance process, based on plausible assumptions, that 


\section{TABLE 1: Sum of Attenuated Erlang Distributions}

\begin{tabular}{llllllll} 
Time Shape $(\boldsymbol{k})$ & $\mathbf{0}$ & $\mathbf{0 . 2 5}$ & \multicolumn{1}{c}{$\mathbf{0 . 5}$} & \multicolumn{1}{c}{$\mathbf{1}$} & $\mathbf{2}$ & $\mathbf{4}$ \\
\hline 1 & 0 & 0.03885704 & 0.07469434 & 0.13823 & 0.238245 & 0.31061 & 0.362969 \\
\hline 2 & 0 & 0.00077529 & 0.00293995 & 0.010581 & 0.034419 & 0.063375 & 0.092798 \\
\hline 3 & 0 & $1.0383 \mathrm{E}-05$ & $7.82 \mathrm{E}-05$ & 0.000555 & 0.003504 & 0.009376 & 0.017 \\
\hline 4 & 0 & $1.0457 \mathrm{E}-05$ & $1.5686-06$ & $2.21 \mathrm{E}-05$ & 0.000274 & 0.001078 & 0.002659 \\
\hline 5 & 0 & $8.4365 \mathrm{E}-10$ & $2.5242 \mathrm{E}-08$ & $7.06 \mathrm{E}-07$ & $1.73 \mathrm{E}-05$ & 0.000101 & 0.000328 \\
\hline 6 & 0 & $5.6766 \mathrm{E}-12$ & $3.9066 \mathrm{E}-12$ & $1.89 \mathrm{E}-08$ & $9.19 \mathrm{E}-07$ & $7.96 \mathrm{E}-06$ & $3.41 \mathrm{E}-05$ \\
\hline 7 & 0 & $3.2755 \mathrm{E}-14$ & $3.9066 \mathrm{E}-12$ & $4.34 \mathrm{E}-10$ & $4.2 \mathrm{E}-08$ & $5.42 \mathrm{E}-07$ & $3.07 \mathrm{E}-06$ \\
\hline 8 & 0 & $1.6543 \mathrm{E}-16$ & $3.9415 \mathrm{E}-14$ & $8.74 \mathrm{E}-12$ & $1.68 \mathrm{E}-09$ & $3.24 \mathrm{E}-08$ & $2.44 \mathrm{E}-07$ \\
\hline 9 & 0 & $7.4284 \mathrm{E}-19$ & $3.5365 \mathrm{E}-16$ & $1.57 \mathrm{E}-13$ & $6.00 \mathrm{E}-11$ & $1.73 \mathrm{E}-09$ & $1.73 \mathrm{E}-08$ \\
\hline 10 & 0 & $3.0026 \mathrm{E}-21$ & $2.8568 \mathrm{E}-18$ & $2.53 \mathrm{E}-15$ & $1.93 \mathrm{E}-12$ & $8.31 \mathrm{E}-11$ & $1.1 \mathrm{E}-09$ \\
\hline 11 & 0 & $1.1034 \mathrm{E}-23$ & $2.0984 \mathrm{E}-20$ & $3.71 \mathrm{E}-17$ & $5.65 \mathrm{E}-14$ & $3.64 \mathrm{E}-12$ & $6.42 \mathrm{E}-11$ \\
\hline 12 & 0 & $3.7176 \mathrm{E}-26$ & $1.4132 \mathrm{E}-22$ & $4.99 \mathrm{E}-19$ & $1.52 \mathrm{E}-15$ & $1.46 \mathrm{E}-13$ & $3.43 \mathrm{E}-12$ \\
\hline Sum & 0 & 0.03964282 & 0.07771408 & 0.149389 & 0.27646 & 0.384549 & 0.47649 \\
\hline Exponential & 0 & 0.03964282 & 0.07771408 & 0.14939 & 0.27646 & 0.384549 & 0.47649
\end{tabular}

A model of the FOD/line maintenance process, here with $P_{\mathrm{R}}=0.5$ and $\lambda_{\text {FOD }}=0.308$, and thus $\lambda_{\text {FOD Removal }}=0.1618$. In general $\lambda_{\text {FOD Removal }}=\left(\lambda_{\text {FOD }} \times P_{\mathrm{R}}\right)$.

provides specific predictions which run counter to the observed behavior. This discrepancy presents an opportunity to identify and test possible explanations to lead us to a better understanding of the FOD plus maintenance process.

\section{FUTURE WORK}

Three routes to resolve this conundrum are open to us, short of challenging the fundamental assumption that FOD incidence and character is independent of time on wing.

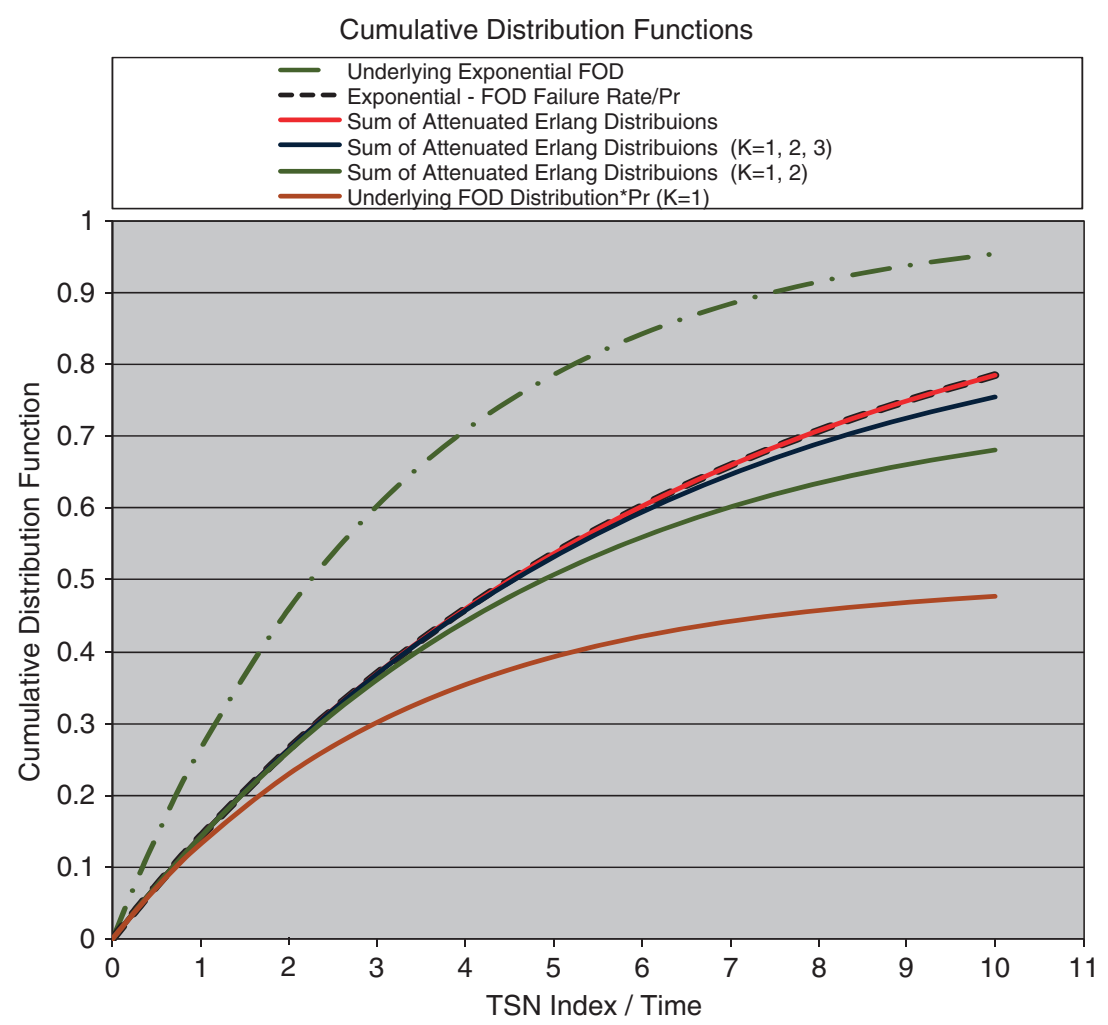

Figure 4: An Illustration of the Attenuated Erlang Distribution Model of FOD Maintenance. Starting with the underlying FOD exponential distribution at top, generate the distribution of FOD removals at the first incidence of FOD, then successively add the removals for second, third, etc. FOD events, which then overlays the exponential distribution for $\lambda_{F O D}$ Removal $=\left(\lambda_{F O D} \times P_{R}\right)$. 
1. Develop and apply plausible models with variable $P_{\mathrm{R}}$. Parametric statistical analysis is in work to evaluate the hypothesis that $P_{\mathrm{R}}$ varies with the number of removals, challenging the assumption of perfect renewal, which may suggest models for $P_{\mathrm{R}}$ dependence on prior FOD incidence.

2. Develop and apply plausible models incorporating variation in $\lambda_{\mathrm{FOD}}$ and $P_{\mathrm{R}}$ for FOD of different origins, and the possibility that $P_{\mathrm{R}}$ is dependent on engine time on wing. (The latter might model the maintainer's reluctance to remove an engine soon after installation, perhaps compensated for by more frequent inspection.)

3. Collection and analysis of detailed line maintenance records would be the most valuable measure; illuminating the underlying FOD frequency and severity, enabling better understanding of the suitability of current maintenance processes and criteria, and enabling informed definition and validation of the models proposed above.

\section{Conclusion}

As Lawless (2003, p. 38) says: "It is important to bear in mind that models only approximate reality, and that in a given situation several models may provide a good description of observed data." Equally, a model is only as valid as the data available to test it.

This work provides an interesting example of the dialectic between parametric statistical data analysis and modeling of the physics (and process in this case) producing the behavior being studied. Although the Erlang/Gamma fit to the data appears more satisfying than the exponential and promises to be a good tool to forecast FOD removals, it conflicts with the characteristics we expect of FOD and the maintenance process.

A simple stochastic model of the FOD/line maintenance process improved our understanding of the FOD/line maintenance process and its implications, reinforcing the question of why the removals data diverges significantly from an exponential distribution. Further elucidating this discrepancy is necessary to provide assurance that engine removal forecasts based on historical data are reliable.

The understanding we sought of the frequency of FOD events resulting in the observed distribution of removals eluded us; we need to collect and analyze line maintenance inspection observations and records of the rework carried out to return the engine to service. This level of detail is expected to yield a better understanding of FOD driven engine removals and improved maintenance forecasting tools, the key to reliability centered maintenance and its benefits.

The simple model of the FOD/line maintenance process developed here has the potential, with elaboration, to further improve our understanding of the observed FOD removal drivers and outcomes and may have applicability to other similar processes in aviation maintenance and other fields.

\section{References}

Anon, "WP 165, intermediate maintenance, cleaning and repair of fan rotor assembly, part no. 5100T63G02." F414-GE-400 Intermediate Maintenance Manual (Volume 1)-Al-F414-MMI-240 (US Navy internal publication), May 1, 2009.

Lawless, J.F., Statistical models and methods for lifetime data, 2nd ed., John Wiley \& Sons, Hoboken, NJ, 2003.

Millar, R.C., "Non-parametric analysis of a complex propulsion system data base." Dissertation, The George Washinģton University, Washinģton, DC, 2007.

Millar, R.C., T.A. Mazzuchi, and S. Sarkani, "Application of non-parametric statistical methods to reliability database analysis." SAE AeroTech (Paper 2007-01-3861), Los Angeles, 2007.

Millar, R.C., T.A. Mazzuchi, and S. Sarkani, "Nonparametric statistical analysis of the reliability of a naval aviation propulsion system," Naval Engineers Journal, Vol. 121, No. 2, pp. 111-121, 2009.

NAVAIR, "NAVAIR 00-25-403: guidelines for the naval aviation reliability-centered maintenance process." July 1, 2005.

US Department of Defense, "The Under Secretary of Defense for Acquisition, Technology and Logistics 
(Kenneth J. Kreig), instruction number 4151.22, 'Condition Based Maintenance Plus (CBM+) for material management,' US Department of Defense, December 2, 2007.

\section{Huthor Biographies}

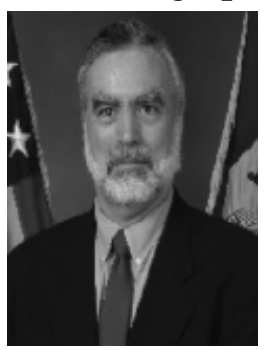

Richard C. Millar is an Associate Professor with the Naval Postgraduate School Department of Systems Engineering, based at the Patuxent River Naval Air Station. He has $35+$ years experience in the design and development of gas turbine engines and their integration with aircraft propulsion and power systems. He has worked in this field at General Electric, United Technologies, Rolls-Royce, Boeing, Lockheed Martin, and BAE Systems before joining NAVAIR in 2003. Dr. Millar has an active research program currently focused on the systems engineering and development of integrated instrumentation/sensor systems for use in the test and evaluation, diagnostics and control of aerospace equipment. Dr. Millar acquired a B.Eng and M.Eng (Carleton) and an MSc in Management (as an MIT Sloan Fellow sponsored by United Technologies) before working for the US Navy. In 2007 be received a DSc in Systems Engineering from The George Washington University with the financial support of NAVAIR.

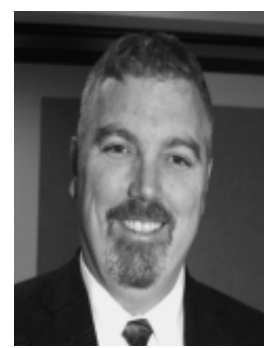

David H. Olwell is Professor of Systems Engineering at the Naval Postgraduate School, and the immediate past department chairman. He is a Fellow of the American Council on Education. 